\title{
Research on Model Establishment of Environment Evaluation of Arctic Route Navigation
}

\author{
Wenlu Bi ${ }^{1, a}$, Tingting Luo ${ }^{1, b}$, Xiaoting Fan ${ }^{1, c}$, Lei Bai ${ }^{1, d}$, Feng Zhang ${ }^{1, e, *}$, Yanfen Gong ${ }^{1, f}$, \\ Jinrong $\mathrm{Li}^{1, \mathrm{~g}}$ \\ ${ }^{1}$ National Marine Data and Information Service, Tianjin, 300171, China; \\ awqubv411@163.com, b55518687@qq.com, '2399133462@qq.com,

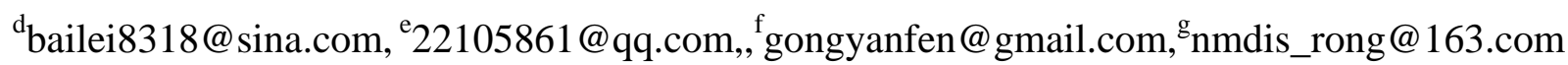 \\ *corresponding author
}

Keywords: Arctic route, Navigation environment, Environment evaluation.

\begin{abstract}
With the global warming in recent years, the ice on the Arctic channel has gradually melted, and the Arctic channel has become the focus of attention in the maritime sector. In order to study the navigation of the Arctic route, this paper builds a model for the navigation environment of the Arctic route, selects the meteorological factors, hydrological factors, geographical factors and transportation factors as the first-class indexes of navigation environment. The weights of the indexes are given by analytic hierarchy process. On the basis of this, the Arctic is unsafe and navigable which could be obtained to provide some references for the relative researchers.
\end{abstract}

\section{Introduction}

In recent years, the ice of Arctic sea was decreasing greatly with the global warming, and the thickness of sea ice has been thinning and the sea ice concentration has been decreasing. The Northeast channel and the northwest channel appeared simultaneously. The Arctic attracted world's attention. For our country, the Arctic waterway has the advantage of distance, especially for the northern port of China, can greatly shorten the voyage, reduce the cost of shipping, increase the shipping market and stimulate economic of China, furthermore, it could also reduce the dependence on the Malacca strait. Meanwhile, the Arctic is rich in natural resources such as oil and mineral resources. The opening of the Arctic route will enable China to establish a resource corridor and increase the opportunities for China's resource trade. Although the Arctic has gradually been opened up, it is still short of the data of Arctic climate, hydrology, channel navigation environment information, and the Arctic navigation experience, the Arctic navigation is very dangerous without the necessary shipping infrastructure. Navigation in the Arctic is greatly affected by the seasons. In winter, almost no ships operate in the arctic. in summer, activities in the Arctic began to perk up, and local cargo ships, dry bulk carriers, and cruise ships began to operate in the area. Even the Arctic temperatures rised as expected, summer and autumn months are prolonged, the regions of Arctic in winter remained largely covered by sea ice and are not navigable. Navigation will still be very seasonal in future. it is the most important to ensure the safety of navigation and prevent environmental pollution in the Arctic route research, it is of great significances of finding out the main influence factors, assign weights, and investigating the environment of the Arctic route to analyze the navigation environment and the development of the Arctic route. 


\section{Index Selection of Environment Evaluation of Arctic Route Navigation}

Meteorological Factors. Air temperature. It mainly has two influences of the temperature on ship navigation. On the one hand, sea water may be frozen at low temperature. The ship's maneuverability will be affected when sea water is frozen on the hull structure, On the other hand, it will affect the living conditions of the crew. Navigation environment is a combination of man, machine and environment, if the crew's living condition is bad, it will inevitably affect the ship's operation.

Visibility. The influence of visibility on navigation environment is remarkable. Visibility of the sea surface is affected by many factors, including sunshine time, rainfall, snowfall, fog, etc. Fog is a major cause of low visibility in the Arctic. In warm months, the most common fog is advection fog. When warm moist air moves horizontally, it cools gradually with the cold air below the dew point temperature, and then gradually cools and forms fog. Another factor affecting Arctic navigation is the lack of color contrast, especially when objects are covered with snow, it will be unable to tell the right distance of objects from their position.

Wind. Arctic wind are often accompanied by storms, and usually there is no hint. Even in windy weather, the speed of wind is rarely exceeds $25 \mathrm{~m} / \mathrm{s}$. In all seasons, the Arctic in the North Atlantic Ocean, Bering Sea and Baffin Bay, Chukotka sea, the activities are often the strongest because of wind and cyclone. The annual speed of wind averaged $6-9 \mathrm{~m} / \mathrm{s}$ on the Pacific coast. In the Atlantic area, the maximum speed of wind can reach $50 \mathrm{~m} / \mathrm{s}$ in winter.

Hydrological Factors. Ocean current velocity. The influence of ocean current and tide on navigation is mainly reflected in the influence of ship handling performance. In general, the greater the water current velocity, the more difficult it is for the ship to navigate, and the possibility of the occurrence of traffic accidents is correspondingly greater. Therefore, currents and tides are important factors affecting the navigation environment. The ocean currents in the Arctic sea route, as the coastal currents of the shallow continental shelf, are dominated by the topographic elements, and flows from west to East. The narrow channel between the islands is marked by tidal currents and strong currents.

Thickness of sea ice. Among the many natural factors affecting the navigation environment of Arctic routes, sea ice is the most influential factor. Sea ice is one of the most important factors that restrict the development of Arctic routes. According to the movement state of sea ice, sea ice can be divided into fixed ice, ice and iceberg. According to the stage of sea ice development, they can be divided into new ice, one-year ice, two-year ice and multi-year ice. On the Arctic route, the main factors affecting navigation are channel, sea ice concentration, sea ice thickness and iceberg distribution.

Geographical Factors. Channel depth. Channel depth refers to the vertical distance from the surface to the bottom of the channel. Channel depth is an important index for channel maintenance, which is generally divided into two kinds, channel maintenance depth and channel standard depth. Channel maintenance depth is the maintenance plan index for waterway maintenance, which is determined by channel maintenance department according to the change of water level, combined with channel change and waterway maintenance capacity. The standard water depth of a channel is the minimum depth need to meet the requirements of a standard ship or fleet for safe navigation during the design of the lowest navigable water level.

Channel curvature. When a ship need bending during the navigation at the channel, it is difficult to operate because it is likely to be affected by channel scale and water flow. It is easy to cause an accident at the bend of the channel. When the ship is sailing on the Arctic route, it is affected by sea ice and there are no fixed routes. Therefore, the channel curvature is also dynamic, mainly due to the influence of water depth, sea ice distribution and partial channel curvature.

Distribution of island. It will increase operational risks of ships in navigating a large number of islands and reefs. This situation is serious in the northwest passage of the northern Canadian islands. Specifically, the barrow Strait, Queen Maud Bay, Fawkes Bay.

Transportation Factors. Port facilities. Port facilities refers to the wharf, breakwater, embankment and revetment, harbor, waterway port, anchorage, Port Road and yard, warehouse, loading and unloading port railway track, mechanical protection facilities and other production 
facilities and affiliated production facilities. When a ship encounters an emergency at sea, it needs to look for a port to take emergency shelter. Most of the ports on the Arctic coast are below 9 meters, which makes it impossible for large vessels to dock in the port. At the same time, there is not enough capacity to repair the ship.

Navigation facilities. There is a geomagnetic storm phenomenon in the Arctic, so it has serious impact on the Arctic Ocean ice navigation. In addition to the navigator, many facilities including log, magnetic compass, radar and other navigation instruments are very limited in the Arctic, it also has great difficulty in the use of landmark positioning, astronomical positioning and radio positioning. In some areas, the communication equipment cannot accept signal synchronous satellite for the navigation of ships as reference and recognition in addition to using iridium phone.

Icebreakers. The icebreaker is used for crushing the ice and opening channel to ensure the frozen port for anchorage or providing navigation service for the ships. Containing rivers icebreaker, lakes icebreaker, harbors or sea ice breaker. it plays important role on maintaining ice waterways unimpeded for navigation, searching and rescuing operations and other functions, therefore, it is very important for the protection of the Arctic route navigation safety.

\section{Model Establishment of Environment Evaluation of Arctic Route Navigation}

Weight Determination. In actual scientific research, such as social phenomena analysis, economic measures, decision-making and scientific or technological evaluation, we often confront the complex factors with the standards of interaction and the lack of quantitative data. In the absence of scientific tools, these are just like a mess in front of us so that we should begin to analyze, let alone getting satisfied evaluation results. The advent of analytic hierarchy process provides us with a scientific tool for addressing such problems. Compared to other decision methods, the method of analytic hierarchy process could avoid the blind pursuit of complex mathematics, and it is concise and easy to use. Its mathematical theory is simple but not perfect, and it is simple to use. It could simplify complex problems. The most important thing is that its evaluation results can match the actual situation. The analytic hierarchy process (AHP) theory is simple and does not require profound theoretical knowledge. The user only needs to have a certain understanding of the decision problem. It has only one step of the real decision process: to enter the judgment matrix without the need for complex scientific calculations. AHP could make quantitative and qualitative analysis. Analytic hierarchy process adopts relative scale, so it can measure subjective and objective, quantitative and non-quantitative factors organically, and combine qualitative and quantitative factors organically. In the view of decision making, analytic hierarchy process regards the problem as a system at first. It studies each component of the system, the relation of each factor and the environment of the system. This approach to the problem helps to make decisions about complex issues. The weights of the indexes of environment evaluation of arctic route navigation calculated from AHP are shown in Table 1. 
Table 1. Index weights of environment evaluation of arctic route navigation

\begin{tabular}{|c|c|c|c|}
\hline First-class indexes & Weight & Second-class indexes & Weight \\
\hline \multirow{3}{*}{ Meteorological factors } & \multirow{3}{*}{0.16} & Air temperature & 0.28 \\
\hline & & Visibility & 0.39 \\
\hline & & Wind & 0.33 \\
\hline \multirow{2}{*}{ Hydrological factors } & \multirow{2}{*}{0.36} & Sea current velocity & 0.42 \\
\hline & & Sea ice thickness & 0.58 \\
\hline \multirow{3}{*}{ Geographical factors } & \multirow{3}{*}{0.13} & Channel depth & 0.29 \\
\hline & & Channel curvature & 0.16 \\
\hline & & Island distribution & 0.55 \\
\hline \multirow{3}{*}{ Transportation factors } & \multirow{3}{*}{0.35} & Port facilities. & 0.55 \\
\hline & & Navigation facilities. & 0.21 \\
\hline & & Icebreakers. & 0.24 \\
\hline
\end{tabular}

Environment Evaluation. An evaluation set is a collection of all the evaluation results that may be made by an object to be evaluated. Evaluation goal of the Arctic route navigation environment is to judge the Arctic navigation and the safety of navigation, therefore the Arctic route navigation environment evaluation set can be partitioned according to the navigation and security level. According to the safety levels, the Arctic route navigation environment could divide into five levels, Safe and navigable, safer and navigable, generally navigable, unsafe and navigable, non navigable. Namely the evaluation set: \{safe navigation, safer navigable, generally navigable, unsafe navigable, not navigable . With global warming, great changes are taking place in the Arctic climate and hydrological environment, furthermore, the Arctic perennial ice, harsh environment, there is not enough observation in the Arctic system, therefore, the data in each index of Arctic route navigation environment evaluation is not applicable in today. In this paper, the expert scoring method is adopted in the evaluation of each index, and the $0 \sim 100$ scale is used in each evaluation. 100 represents the best navigable environment, the safest one, and the 0 represents the worst. Set up the corresponding relation between evaluation set and score. We concluded that the navigation environment of the Arctic route is unsafe, but navigable. In this paper, the factors affecting the navigation environment of the Arctic channel are analyzed, the index evaluation system is established, and the navigation environment of the Arctic is evaluated, which provides reference for the utilization and supervision of the Arctic channel.

\section{Conclusion}

With the global warming, the Arctic sea ice accelerates ablation, and the Arctic channel has attracted more and more world's attention due to its great advantages in the voyage. This paper analyzes the characteristics of navigation environment of Arctic channel, and builds an evaluation model for navigation environment of Arctic route. By means of analytic hierarchy process (AHP), we determine the weights of the indexes at all levels, and on this basis, we get the overall evaluation of the navigation environment of the Arctic channel. With the Arctic warming, the Arctic's hydrological environment will be more conducive to navigate.

\section{Acknowledgments} fund.

This work was financially supported by National Marine Data and Information Service youth 


\section{References}

[1] Li Zhenfu, Yan Li, Xu Mengqiao, Liu Baihe, Ma Shumeng, Evaluation of Arctic route's navigation environment [J]. Computer Engineering and Applications, 2013, 49 (1): 249-253.

[2] Li Zhenfu, Ren Yanyang, Ma Shumeng, Tang Xiaowen, Li Yi, Li He, The Evaluation of Arctic Route Navigation Environment by Blind Number Model[J]. Journal of Jimei University (Natural Science), 2013, 18(3): 185-191.

[3] Shu Chi, Bing Lei, Sun Fangfang, Li Zhenbo, Survey on the navigation situation of the Arctic on the base of satellite remote sensing technology [J]. China Maritime Safety, 2016(1): 53-55.

[4] Wang Luo, Zhao Yue, Liu Jianmin, Han Shuqin, China’s First Trans-Arctic Voyage and Related Expectations [J]. Chinese Journal of Polar Research, 2014, 26(2): 276-284. 